\title{
Patient Portal Use Near the End-of-Life
}

$\mathrm{J}$ Gen Intern Med

DOI: $10.1007 / \mathrm{s} 11606-020-06333-9$

(c) Society of General Internal Medicine 2021

\section{INTRODUCTION}

Patient portals ${ }^{1}$ are secure websites for personal health information and tailored resources linked to a patient's electronic health record (EHR). Approximately 52\% of patients access their portal ${ }^{2}$ although use in seriously ill populations is unknown, as is use by caregivers. ${ }^{3,4}$ Understanding current portal use can inform strategies to improve patient outcomes and facilitate caregiver activities during end-of-life care. We described patient and caregiver portal use patterns among adults with serious illness nearing endof-life within Kaiser Permanente Colorado (KPCO), where $63 \%$ of the adult population is registered for the My Health Manager (MHM) portal.

\section{METHODS}

Study population inclusion criteria were (1) seriously ill patients (defined by KP's proprietary "Care Group" algorithm), $\geq 18$ years of age, who were registered in MHM, and who died between January 1, 2016 and June 30, 2019; and (2) caregivers, $\geq 18$ years of age, of these patients registered for a proxy portal account. We extracted data from KPCO's EHR and MHM portal databases. Data included patient characteristics and portal use metrics (discrete days of use, features accessed) summarized monthly over the 12-month study period prior to death. To examine changes in portal use over the study period, models included an unadjusted linear trend of the days of any portal usage by month using a generalized estimating equation (GEE) Poisson model with a log link and an autoregressive correlation structure of order 1. All analyses were conducted using SAS statistical software, version 9.4 (SAS Institute Inc., Cary, NC). Study procedures were approved by the KPCO Institutional Review Board.

\section{RESULTS}

We identified 6517 seriously ill patients with MHM registrations; 163 of these patients had proxy caregivers with a MHM account (Table 1). Patient users were 77 years of age mostly categorized as frail and White, and caregivers were predominantly female. Average days of portal use over the last 12 months of life among patients were 42.4 days and $<1$ day among their caregivers. Number of days used significantly increased by about $0.7 \%$ per month from 12 months to 1 month prior to death $(95 \%$ CI: $0.4-1.0 \% ; p$ value $<.0001)$ and peaked with use of most portal features 3 months prior to the patient's death (Fig. 1).

\section{DISCUSSION}

We observed an increase in portal use 3 months prior to the patient's death. Average use in this cohort was high in comparison to previous research on portal use patterns that estimated between 18.5 days $^{5}$ and 27.2 days $^{6}$ of portal use in a 12-month period. Use increases were found among Care Team Information, Medical Record, and New Features (e-visits and chat) while Health Management Tools and Cost-Coverage use remained low. This 
Table 1 Characteristics of My Health Manager (MHM) Users and Utilization

\begin{tabular}{|c|c|c|}
\hline & Patients $(N=6517)$ & Caregiver/proxy $(N=163)$ \\
\hline \multicolumn{3}{|l|}{ Characteristic } \\
\hline $\mathrm{Age}^{\mathrm{a}}$, mean $(\mathrm{SD})$ & $76.7(13.7)$ & $61.0(13.0)$ \\
\hline Female $(\%)$ & 49.9 & 77.9 \\
\hline \multicolumn{3}{|l|}{ Kaiser Care Group (\%) } \\
\hline Group 2: Serious Chronic Conditions & 15.3 & \\
\hline Group 3: Advanced Illness & 21.1 & \\
\hline Group 4: Frailty or End-of-Life & 63.6 & \\
\hline \multicolumn{3}{|l|}{ Race $(\%)$} \\
\hline American Indian/Alaska Native & $<1.0$ & 1.2 \\
\hline Asian & 1.4 & 2.5 \\
\hline Black or African American & 2.5 & $<1.0$ \\
\hline Native Hawaiian/other Pacific Islander & $<1.0$ & $<1.0$ \\
\hline White & 83.6 & 81.6 \\
\hline Unknown/other & 11.6 & 13.5 \\
\hline Hispanic $(\%)$ & 7.2 & 14.1 \\
\hline \multicolumn{3}{|l|}{ MHM utilization } \\
\hline Days portal was used in 12 months approaching death, mean (SD) & $42.4(40.3)$ & $.71(7.1)$ \\
\hline \multicolumn{3}{|l|}{ Days portal feature was used in 12 months, mean (SD) } \\
\hline Patient-Provider Communication ${ }^{\mathrm{b}}$ & $20.8(25.6)$ & $.43(4.4)$ \\
\hline Visits and Appointment Scheduling & $13.6(19.1)$ & $.29(3.5)$ \\
\hline Labs and Test Results & $11.4(16.8)$ & $.19(2.2)$ \\
\hline Recently Added Features ${ }^{\mathrm{c}}$ & $11.2(19.0)$ & $.16(1.9)$ \\
\hline Medications & $9.4(16.2)$ & $.11(1.7)$ \\
\hline Medical Record ${ }^{\mathrm{d}}$ & $4.6(8.5)$ & $.08(1.3)$ \\
\hline Care Team Information ${ }^{\mathrm{e}}$ & $10.8(12.0)$ & $0(0)$ \\
\hline Health Management Tools ${ }^{\mathrm{f}}$ & $1.4(3.0)$ & $.02(.36)$ \\
\hline Cost and Coverage & $.9(2.4)$ & $.01(.13)$ \\
\hline
\end{tabular}

${ }^{a}$ Patient age was calculated at date of death. Caregiver age calculated at the end of the study period

SD, Standard deviation

${ }^{b}$ Provider Communication includes secure messaging with physicians, nurses, and other support assistants about care, logistics, lab results, etc.

${ }^{c}$ New Features included e-visits and chat functions for non-emergent questions

${ }^{d}$ Medical Record included health summary lists, ongoing conditions, eyewear prescription, allergies, and immunization records

${ }^{e}$ Care Team Information included list of all care providers linked to profiles about the providers and their clinics

${ }^{{ }^{\prime} H e a l t h}$ Management Tools included health guides, living healthy tools, videos, health encyclopedia, connects with classes, support groups, and wellness coaching

suggests patients and caregivers may have been seeking a comprehensive understanding of the patient's health status and trying to communicate with their health care team during a time when access to ambulatory services may be limited by declining function. Previous research highlights the benefit of the portal for improving patientprovider communication. ${ }^{7,8}$ A spike in portal usage among seriously ill patients could be used as an indicator for providers to reach out to patients and caregivers or trigger a palliative care consult.

This is one of the first studies to examine portal use near the end-of-life. There are several limitations. The study was conducted in a single health system in which patients frequently use the portal system and all were insured. Our sample was primarily White and nonHispanic, which is not representative of Colorado's racial distribution, limiting our ability to generalize these findings to a more diverse population. We were unable to capture caregivers who logged in using patient credentials.

Patients with serious illness and their caregivers are using portals near the end-of-life. Use patterns suggest that as the patient approaches death, portal use increases. Future research is needed to explain this trend and explore how portals may serve as indicators for identifying and addressing end-of-life care needs. 


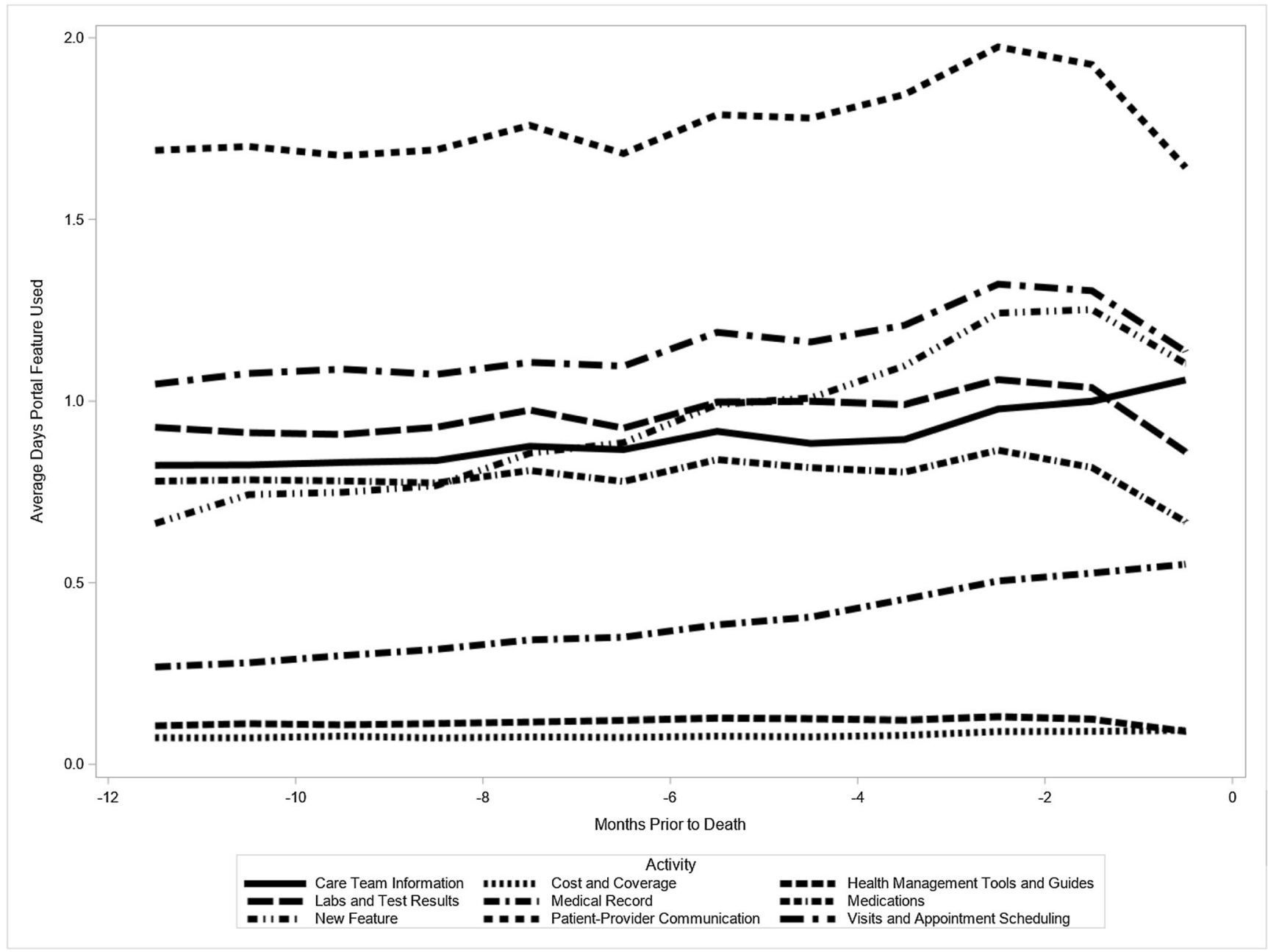

Figure 1 Patient portal feature use during the last 12 months of life. Average number of days MHM was used by feature increased over the last 12 months of life. Number of days features was used per person per month ranged from 0 to 31 days (interquartile range $0-1$ days).

Jennifer Dickman Portz, PhD, MSW ${ }^{1,2}$

J. D. Powers, $\mathrm{MS}^{2}$

M. Baldwin, $B A^{2}$

David B. Bekelman, $M D, M P H^{1,3}$

Alejandra Casillas, $M D^{4}$

Jean S. Kutner, MD, MSPH ${ }^{1}$

Elizabeth Bayliss, $M D, M P H^{2,5}$

${ }^{1}$ Division of General Internal Medicine, Department of Medicine, University of Colorado School of Medicine,

Aurora, CO, USA

${ }^{2}$ Institute for Health Research, Kaiser Permanente Colorado,

Aurora, CO, USA

${ }^{3}$ Department of Medicine, Department of Veterans Affairs, Eastern Colorado Health Care System,

Aurora, CO, USA

${ }^{4}$ Division of General Internal Medicine and Health Services Research, David Geffen School of Medicine, UCLA,

Los Angeles, CA, USA
${ }^{5}$ Department of Family Medicine, University of Colorado School of Medicine,

Aurora, CO, USA

Corresponding Author: Jennifer Dickman Portz, PhD, MSW; Division of General Internal Medicine, Department of Medicine, University of Colorado School of Medicine Aurora, CO, USA (e-mail: jennifer.portz@cuanschutz.edu).

Funding This project is supported by the Palliative Care Research Cooperative Group funded by National Institute of Nursing Research (U2CNR01463) and a career development award from the National Institute on Aging (K76AG059934).

\section{Compliance with Ethical Standards:}

Study procedures were approved by the KPCO Institutional Review Board.

Conflict of Interest: The authors declare that they do not have a conflict of interest. 


\section{REFERENCES}

1. HealthIT. What is a patient portal? URL: https://www.healthit.gov/faq/ what-patient-portal. Accessed 17 July 2020. https://www.webcitation. org/6yM3Tstn6

2. Patel V, Johnson C. Individuals' Use of Online Medical Records and Technology for Health Needs. Office of the National Coordinator for Health Information Technology; 2018. https://www.healthit.gov/ sites/default/files / page/2018-03/HINTS-2017-Consumer-DataBrief-3.21.18.pdf. Accessed 17 July 2020

3. McCleary NJ, Greenberg TL, Barysauskas CM, et al. Oncology Patient Portal Enrollment at a Comprehensive Cancer Center: A Quality Improvement Initiative. J Oncol Pract. 2018;14(8):e451-e461. https://doi.org/10. 1200/JOP.17.00008

4. Ammenwerth E, Schnell-Inderst P, Hoerbst A. The Impact of Electronic Patient Portals on Patient Care: A Systematic Review of Controlled Trials. J Med Internet Res. 2012;14(6):e162. https://doi.org/10.2196/jmir.2238
5. Jones JB, Weiner JP, Shah NR, Stewart WF. The Wired Patient: Patterns of Electronic Patient Portal Use Among Patients With Cardiac Disease or Diabetes. J Med Internet Res. 2015;17(2):e42. https://doi.org/10.2196/ jmir.3157

6. Riippa I, Linna M, Rönkkö I, Kröger V. Use of an Electronic Patient Portal Among the Chronically Ill: An Observational Study. J Med Internet Res. 2014;16(12):e275. https://doi.org/10.2196/jmir.3722

7. Reed ME, Huang $\mathbf{J}$, Brand $\mathbf{R}$, et al. Communicating Through a Patient Portal to Engage Family Care Partners. JAMA Intern Med. 2018;178(1):142. https://doi.org/10.1001/jamainternmed.2017.6325

8. Pho KK, Lu R, Gates S, et al. Mobile Device Applications for Electronic Patient Portals in Oncology. JCO Clin Cancer Inform. 2019;3(101708809):1-8. https://doi.org/10.1200/CCI.18.00094

Publisher's Note Springer Nature remains neutral with regard to jurisdictional claims in published maps and institutional affiliations. 